\title{
The Psychophysiology OF LOW BACK PAIN
}

\section{Author: $\quad$ Adams Nicola (1997) \\ Published by: Churchill Livingstone, ISBN $044305259 \mathrm{X}$ with a contribution by Douglas Taylor.}

$\mathrm{T}$ his is a very interesting book which will prove useful especially to those who deal with patients with chronic pain, and particularly chronic low back pain (LBP). It is certainly true to its stated purpose, viz to enhance the understanding of the interactions of the factors involved in LBP and to suggest ways of expanding and reinforcing existing practices by presenting neurophysiological, biochemical and psychological. aspects of pain, as well as ways of assessing and modulating the pain experience. A chapter is spent on each of these aspects. The author writes from her own clinical experience and extensive reading and study, which goes much wider than "standard" physiotherapy and expresses her belief in accurate diagnosis, early intervention and appropriate consideration of the psychological factors involved in pain. She particularly stresses the importance of the psychological quali- ties of the patient-practitioner intervention, which can be major mediators for improvement and devotes a whole chapter to discussing this. As physiotherapists we have known about this for generations, anecdotally, and it is useful to have it confirmed scientifically and well referenced.

Douglas Taylor contributes to the two chapters describing and pointing out limitations of a number of psychological models of chronic pain, as well as assessment, including psychological assessment. Some shortened psychological assessment forms/scales that will be useful to physiotherapists, are produced in full in the text and are fully referenced for those who wish to acquire more information. However, some of the newer tests, for example for anxiety, self-esteem, health behaviour and coping strategies, that could be useful in dealing with patients with chronic pain, are mentioned but unfortunately not referenced.

Some of the recommended psychological and psychophysiological techniques, such as progressive relaxation combined with correct breathing, autogenic training, cognitive and cognitive- behavioural techniques (the latter two used mainly by psychologists), psychotherapy and alternative techniques (yoga, meditation, t'ai chi and chi gong) are described, some in more detail than others, and again are well referenced. In keeping with the author's wide perspective on the treatment of patients with pain, the multidisciplinary and back pain management approach is recommended. The Wirral Back Pain Rehabilitation Programme, with very specific outcome measurements, is described in detail and could be useful to those who wish to set up similar programmes.

The text is easy to read and supported by an extensive list of references, which includes some classical ones from the 1930's - 1950's, the bulk from 1970 1989 and a good number from 1990 1996. Some of the items contained in the glossary are rather obvious, whilst others introduce what could be new concepts to physiotherapists. A specialist text, recommended for most physiotherapists, as most have to deal with LBP and chronic pain during their career.

NARINA GILDER 